Int. J. Dev. Biol. 52: 1043-1050 (2008)

doi: $10.1387 / \mathrm{ijdb} .082608 \mathrm{mb}$

\title{
Origin and proliferation of blastema cells during regeneration of Drosophila wing imaginal discs
}

\author{
MANEL BOSCH ${ }^{1}$, JAUME BAGUÑ ${ }^{1}$ and FLORENCI SERRAS ${ }^{*}, 1,2$ \\ ${ }^{1}$ Departament de Genètica, Facultat de Biologia, Universitat de Barcelona and \\ ${ }^{2}$ Institut de Biomedicina de la Universitat de Barcelona (IBUB), Barcelona, Spain
}

\begin{abstract}
Following a period of neglect, there has been a resurgence of interest in Drosophila imaginal discs as a model with which to analyze the relationships between growth and pattern formation during regeneration. To broaden our understanding of this process, we used cell lineage techniques to trace the origin of blastema cells and the early and late boundaries of the blastema in regenerating 3/4 wing disc fragments, examined the distribution of S-phase, mitotic and dead cells, and undertook clonal analysis to determine the topology of cell proliferation and its relationship to pattern formation. Using lineage tagging with the JNK phosphatase puckered (puc), we demonstrate that a substantial number of blastema cells arise from cells in which JNK is activated. Furthermore, we show that DNA synthesis and mitosis are activated well before wound healing is completed, in a region where the JNK pathway is activated; later, DNA synthesis and mitosis are observed in scattered cells throughout the blastema. Finally, clonal analysis shows a close relationship between the size and shape of clones and disparities in the positional values of the apposed surfaces.
\end{abstract}

KEY WORDS: regeneration, imaginal disc, cell proliferation, blastema, pattern formation

\section{Introduction}

Regeneration of lost structures in animals is achieved either by local stimulation of cell proliferation (epimorphosis; Morgan, 1901) or through cell respecification without cell division (morphallaxis; Morgan, 1901). Surgical removal of normal cell neighbors and apposition of new neighbors as a result of wound healing leads to contact between cells that were previously separated. As a result, a discontinuity in positional information is generated (Wolpert, 1969) that may induce local cell proliferation (French et al., 1976). Newly reconstructed tissues will adopt intermediate positional values to smoothen the discontinuities in the distribution of positional information.

This framework, encapsulated among others in the polar coordinate model of pattern formation (French et al., 1976), was tested in regenerating imaginal discs of Drosophila, analyzing patterns of cell proliferation (Dale and Bownes, 1980) by incorporation of ${ }^{3} \mathrm{H}$-thymidine (Adler, 1984, Bryant and O'Brochta, 1986, Dale and Bownes, 1980, Dale and Bownes, 1981, Kiehle and Schubiger, 1985, O'Brochta and Bryant, 1987) or bromodeoxyuridine (Bryant and Fraser, 1988) into S-phase cells and flow cytometry (Fain and Alvarez, 1987). Those studies led to the following conclusions: (1) in most cases DNA synthesis but not mitosis precedes the completion of healing (except in the leg primordium, as reported in Kiehle and Schubiger, 1985); (2) the regenerative blastema is defined by clusters of S-phase labeled cells spanning width ranges of 25-75 $\mu \mathrm{m}$ (equivalent to 8-25 cell diameters); (3) the time to complete regeneration is roughly proportional to the amount of tissue removed-hence, larger discontinuities in positional value produce larger and more persistent blastemas than do smaller discontinuities; and (4) patterns of proliferation are more consistent with averaging models, in which proliferation begins close to the wound and later spreads throughout the blastema, than sequential models, in which proliferation remains localized within a thin strip of blastema cells. Following those studies, regeneration of Drosophila imaginal discs has continued to be viewed as a clear-cut epimorphic process in which cells close to the wound proliferate and form a blastema from which new cells, produced by intercalary proliferation, restore the lost pattern and positional continuity.

Despite these advances, key questions remain unexplored

Abbreviations used in this paper:JNK, Jun N-terminal kinase ; puc, puckered.

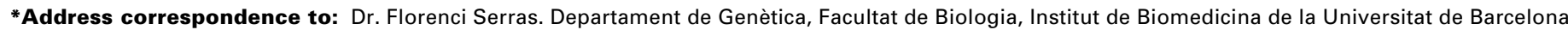
(IBUB), Universitat de Barcelona, Diagonal 645, Barcelona, Spain. e-mail: fserras@ub.edu
} 
and several issues continue to be unresolved. For example, the extent of the tissue from which cells are recruited to proliferate and form the blastema is unknown, and it is not clear whether late blastema boundaries correspond to the early wound edges. Also, whether the topology of cell proliferation and the orientation of mitosis within the blastema follows similar patterns as seen during imaginal disc development is unknown.

In this study we analyzed cell lineage to determine the origin and boundaries of the blastema in relation to the area of cell proliferation. We then analyzed the main parameters of blastema

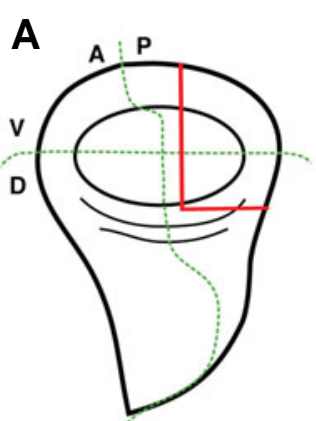
dynamics (DNA synthesis, cell proliferation, and cell death) in wing imaginal disc fragments at different stages of regeneration and used clonal analysis to monitor the topology of cell proliferation and provide insights into how re-patterning is achieved. Cell lineage tracing revealed that most blastema cells arise from cells at the wound bound- ary. Moreover, as already reported by Kiehle and Schubiger (1985) and Mattila etal. (2005), we found that both DNA synthesis and mitosis begin along wound areas prior to completion of wound closure. Later, DNA synthesis and cell division increase within the growing blastema while remaining very low in the rest of the disc.

\section{Results}

\section{Origin and boundaries of the regenerating blastema}

To explore the origin of cells forming the blastema and to define more precisely the extent and boundaries of the blastema, we took advantage of the expression of the JNK phosphatase puckered (puc) in several rows of cells at the edges of the wound (Bosch et al., 2005, Lee et al., 2005) using a cell lineage-tracing method (Weigmann and Cohen, 1999). We lineage tagged cells at the puc-positive wound area using puc-Gal4 to direct expression of the FLP recombinase using a UAS-FLP construct. In regenerating discs from larvae carrying puc-Gal4, UAS-FLP, UAS-GFP and Act5c-FRT-stop-FRT-lacZ, the FLP recombinase is expressed in cells expressing puc-Gal4 and excision of the
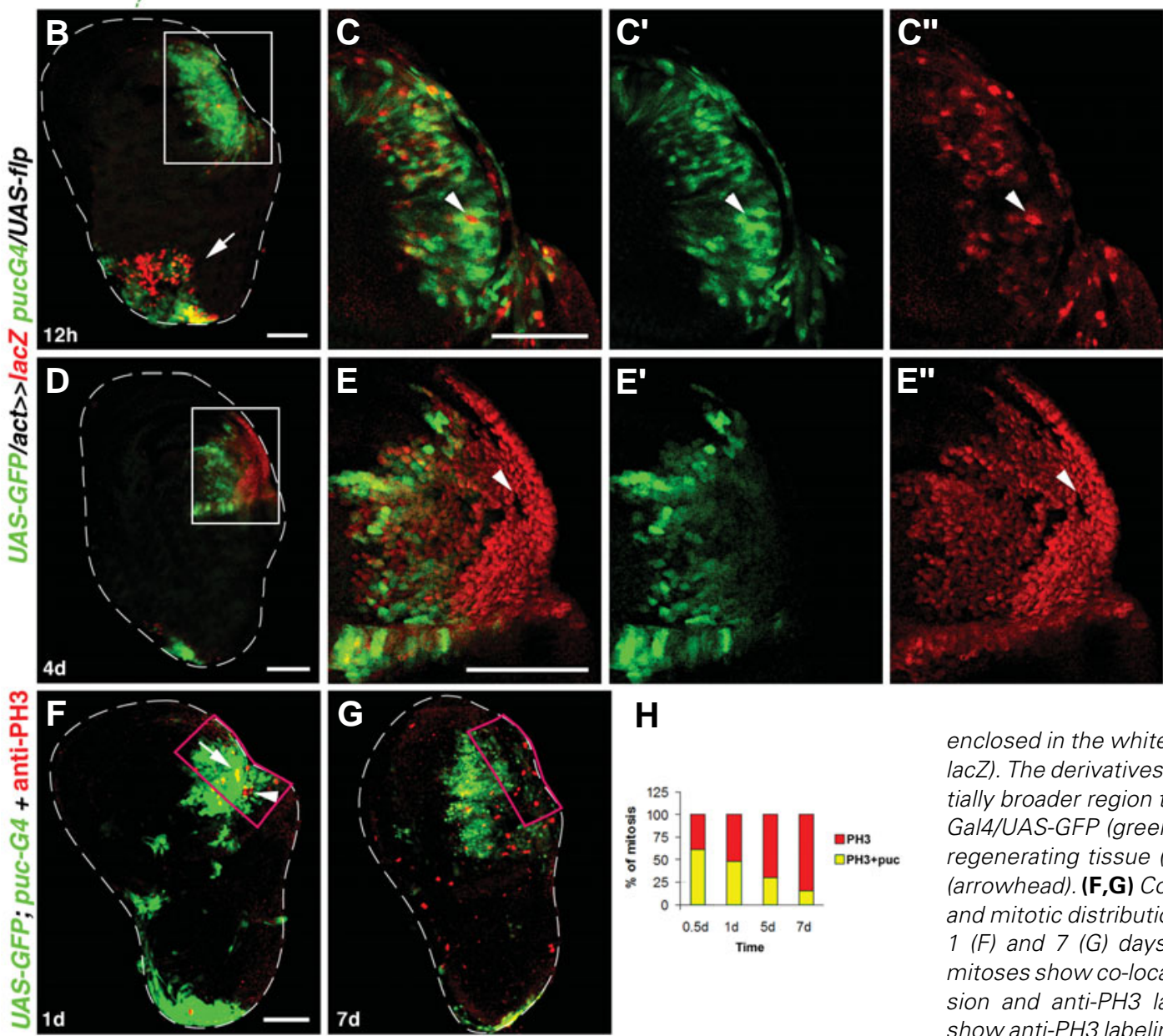

Fig. 1. Lineage tracing of pucGal4 in the blastema of 3/4A regenerating fragments. (A) Diagram of a wing imaginal disc showing the cut used in all experiments (red line). Anteoposterior (AP) and dorsoventral (DV) compartmental boundaries are shown as dotted lines. $(\mathbf{B}, \mathbf{C})$ Blastema of a UASGFP/Act5c>stop>lacZ; puc-Gal4/ UAS-FLP disc at $12 \mathrm{~h}$ of regeneration. Whole wing disc $(B)$ and detail of the blastema enclosed in the white rectangle $(C$, merged; $C^{\prime}$, GFP; $C^{\prime \prime}$, lacZ). In this early stage of regeneration most of the cells express GFP (green) and lacZ (red). Few cells (arrowhead) express lacZ and no or low levels of GFP. (D,E) Blastema of a UAS-GFP/Act5c>stop >lacZ; puc-Gal4/UAS-FLP disc at 4 days of regeneration. Whole wing disc (D) and detail of the blastema H

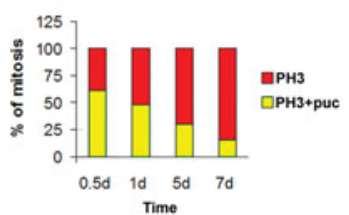
enclosed in the white rectangle (E, merged; $E^{\prime}$, GFP; $E^{\prime \prime}$, lacZ). The derivatives of puc-Gal4 (red) occupy a substantially broader region than the cells that still express pucGal4/UAS-GFP (green). Note that red cells fill the entire regenerating tissue (blastema) except for some clones (arrowhead). (F,G) Comparison of puc expression (green) and mitotic distribution (red) between 3/4A fragments at 1 (F) and 7 (G) days of regeneration. At 1 day, some mitoses show co-localization (yellow dots) of puc expression and anti-PH3 labeling (red), although some only show anti-PH3 labeling (red dots). At 7 days, mitoses are mainly located in the blastema (red dots), distal to the puc expression belt (green), although some mitotic cells (yellow and red) still occur in the blastema boundary. (H) Percentage of mitoses, measured within the Exp $P$ area (magenta rectangles in $F$ and $G$ ) colocalizing with puc expression (yellow bars) or not (red bars) at 0.5, 1, 5 and 7 days of regeneration. Note the decreasing percentage of mitotic cells expressing puc (yellow bars) as regeneration proceeds. Arrow in (B) indicates the endogenous puc expression, which is normally found at the stalk of the notum. Scale bar, $50 \mu m$. 
CONTROL Oh

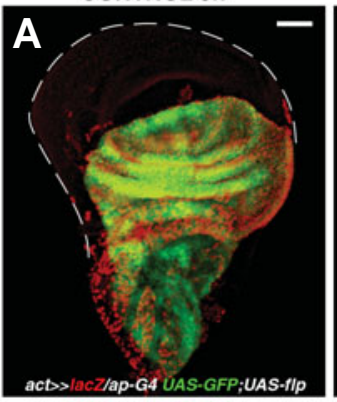

EXPERIMENTAL 7d

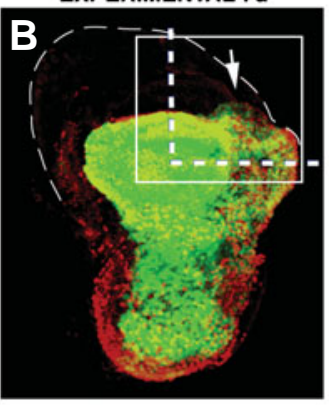

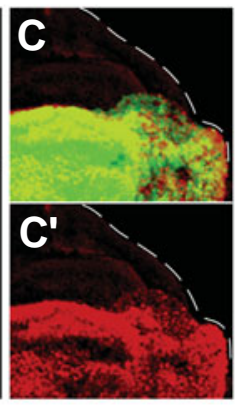

Fig. 2. Lineage analysis of the progeny of ap-Gal4 cells in blastemas of 3/4A fragments to test the contribution of opposing wound edges during regeneration. Confocal sections through Act5c-FRT-stop-FRTlacZlap-Gal4 UAS-GFP;UAS-FLP discs at 7 days of regeneration. (A) Control uncut disc, showing that ap lineage-tagged expression (red) and the actual ap expression (green) co-localize in all cells of the dorsal compartment. (B) A 7-day regenerating disc. White dashed thick line indicates the approximate cutting edges. $\left(C, C^{\prime}\right)$ Higher magnification view of the white square shown in (B). (C) Red and green channels; (C') red channel. Note that the progeny of ap-expressing cells (red) only contribute to the regenerating dorsal compartment. White dashed thin lines delineate the contour of the discs. Scale bar, $50 \mu \mathrm{m}$.

FLP-out stop cassette from the inactive reporter construct generated an active Act5c>lacZ tag. After excision, reporter gene expression is regulated by the actin promoter and is clonally inherited by all the progeny of puc-Gal4 expressing cells in which the recombination event took place. The expression of lacZ is thereafter independent of Gal4.

During the first day of regeneration, most of the lacZ-expressing cells were within the GFP domain. However some lacZ cells contained low GFP, suggesting that cells derived from the blastema began the reconstruction of the missing tissue (Fig. $1 \mathrm{~B}, \mathrm{C}$ ).

At 4 days of regeneration (Fig. $1 \mathrm{D}, \mathrm{E}$ ), more cells expressing lacZ (red) filled most of the regenerated tissue. The puc-Gal4 domain (GFP labeled) delineated a complementary narrower proximal domain. This GFP domain corresponds to puc-Gal4expressing cells from the original wound margin. Therefore, most blastema cells are lineage tagged with lac $Z$ and hence derived from puc-Gal4 positive cells at the wound edge of early stages of regeneration. Interestingly, some regenerated cells lacked lac $Z$ labeling (see arrowhead in Fig. 1E); these cells most likely correspond to progeny of cells of the wound edges that do not express puc (Bosch et al., 2005). Similar results were also found in discs analyzed 7-days after injury (not shown).

To further examine the contribution of pucexpressing cells to blastema formation, we measured the number of puc-GFP-positive and puc-GFP-negative cells undergoing mitosis in the regenerating area of UAS-GFP; puc-Gal4 discs at 0.5, 1, 5 and 7 days of regeneration ( $n=10$ discs). At 0.5 days, when pucexpression is maximal and covers the entire wound area, $60 \%$ of mitotic cells were puc-GFP positive, while $40 \%$ of cells were not. Later, the percentage of mitotic cells that were puc-GFPpositive decreased as regeneration proceeded (Fig. $1 \mathrm{~F}-\mathrm{H}$ ).

Finally, to test whether both wound edges contribute equally to blastema formation, we used a FLP-out cassette activated by an ap-Gal4 driver in the D compartment. Discs carrying Act5c-FRTstop-FRT-lacZ/ap-Gal4 UAS-GFP,UAS-FLP were cut into 3/4A fragments and cultured for 7 days. Our results showed that lacZexpressing cells filled part of the blastema and lacZ-negative cells filled the rest (Fig. 2), likely meeting at the dorso-ventral compartmental boundary. The restricted localization of $\beta$-galactosidase (red) in the ap-Gal4 UAS-GFP (green) domain of regenerated discs implies that the derivatives of the dorsal compartment wound edge contribute exclusively to the dorsal part of the regenerated structure and therefore that both blastema edges contribute to regeneration.

\section{Spatial and temporal dynamics of cell proliferation in regen- erating discs}

To analyze the temporal and spatial pattern of cell proliferation, we measured the number and distribution of S-phase cells after BrdU incorporation and of mitotic cells labelled with PH3. Surprisingly, when analyzed at $12 \mathrm{~h}$ after implantation, cut discs had halted proliferation. Also, intact early third instar discs, which would normally undergo several rounds of cell division, stopped proliferation after implantation. This indicates that cutting and implantation already has an effect within the first $12 \mathrm{~h}$ of implantation. However, after the first $12 \mathrm{~h}$ proliferation is resumed.

The dynamics of BrdU incorporation are summarized in Fig. 3K. Overall, the average number of BrdU-labeled cells was always higher at the Exp $P$ domain (regenerating area) than in other areas (Fig. 3A-C). From 0.5 days on, the number of BrdUlabeled cells increased steadily in the Exp $P$ area, peaking at 2 days. Then, the number of labeled cells decreased steadily to

\section{TABLE 1}

AVERAGE NUMBER \pm STANDARD ERROR OF S PHASE, MITOTIC AND DEAD CELLS IN CONTROL AND EXPERIMENTAL 3/4A WING DISC FRAGMENTS

\begin{tabular}{ccccccc} 
& \multicolumn{6}{c}{ S Phase } \\
\cline { 2 - 7 } Time & $\mathbf{N}_{\mathrm{C}}$ & $\mathbf{C T R L} \mathbf{P} \pm \mathrm{SE}$ & $\mathbf{C T R L} \mathbf{A} \pm \mathrm{SE}$ & $\mathbf{N}_{\mathrm{E}}$ & EXP P \pm SE & EXP A \pm SE \\
\hline $0.5 \mathrm{~d}$ & 10 & $7.9 \pm 1.4$ & $7.2 \pm 2.1$ & 8 & $20.4 \pm 3.5$ & $9.8 \pm 2.1$ \\
$1 \mathrm{~d}$ & 8 & $6.3 \pm 1.1$ & $7.1 \pm 1.4$ & 9 & $61.4 \pm 16.6$ & $14.0 \pm 7.1$ \\
2d & 9 & $8.0 \pm 2.0$ & $7.4 \pm 1.2$ & 10 & $72.0 \pm 10.7$ & $17.2 \pm 4.3$ \\
$3 \mathrm{~d}$ & 14 & $2.9 \pm 0.9$ & $4.6 \pm 1.1$ & 9 & $17.4 \pm 2.4$ & $2.4 \pm 1.0$ \\
$5 \mathrm{~d}$ & 9 & $0.8 \pm 0.2$ & $0.7 \pm 0.4$ & 9 & $9.1 \pm 2.1$ & $1.4 \pm 0.3$ \\
$7 \mathrm{~d}$ & 8 & $1.5 \pm 0.4$ & $2.4 \pm 0.9$ & 9 & $8.7 \pm 3.1$ & $1.6 \pm 0.4$ \\
\hline
\end{tabular}

\begin{tabular}{ccccccc} 
& \multicolumn{6}{c}{ M Phase } \\
\cline { 2 - 7 } Time & $\mathbf{N}_{\mathrm{C}}$ & $\mathbf{C T R L} \mathbf{P} \pm \mathrm{SE}$ & $\mathrm{CTRL} \mathbf{A} \pm \mathrm{SE}$ & $\mathbf{N}_{\mathrm{E}}$ & EXP P \pm SE & EXP A \pm SE \\
\hline $0.5 \mathrm{~d}$ & 9 & $8.4 \pm 1.8$ & $10.2 \pm 2.8$ & 8 & $25.6 \pm 3.6$ & $8.5 \pm 2.1$ \\
$1 \mathrm{~d}$ & 9 & $1.6 \pm 0.5$ & $3.6 \pm 0.8$ & 9 & $11.6 \pm 2.1$ & $3.6 \pm 1.6$ \\
$2 \mathrm{~d}$ & 8 & $1.4 \pm 0.6$ & $2.3 \pm 1.1$ & 9 & $17.2 \pm 3.3$ & $2.2 \pm 0.6$ \\
$3 \mathrm{~d}$ & 8 & $5.6 \pm 0.8$ & $5.0 \pm 0.8$ & 9 & $24.0 \pm 2.9$ & $5.6 \pm 1.2$ \\
$5 \mathrm{~d}$ & 9 & $4.3 \pm 1.1$ & $5.0 \pm 1.6$ & 9 & $15.9 \pm 4.3$ & $5.3 \pm 1.0$ \\
$7 \mathrm{~d}$ & 10 & $11.0 \pm 1.9$ & $10.2 \pm 3.5$ & 8 & $9.1 \pm 2.9$ & $8.0 \pm 2.7$ \\
\hline
\end{tabular}

\begin{tabular}{ccccccc} 
& \multicolumn{6}{c}{ Cell Death } \\
\cline { 2 - 7 } Time & $\mathbf{N}_{\mathbf{C}}$ & CTRL P \pm SE & CTRL A \pm SE & $\mathbf{N}_{\mathrm{E}}$ & EXP P $\pm S E$ & EXP A $\pm S E$ \\
\hline $0.5 \mathrm{~d}$ & 8 & $4.3 \pm 1.9$ & $5.3 \pm 1.8$ & 11 & $11.1 \pm 2.7$ & $5.0 \pm 1.2$ \\
$1 \mathrm{~d}$ & 10 & $16.1 \pm 4.2$ & $9.2 \pm 1.9$ & 8 & $18.1 \pm 3.6$ & $18.1 \pm 4.9$ \\
$2 \mathrm{~d}$ & 8 & $15.5 \pm 1.8$ & $11.0 \pm 1.4$ & 8 & $17.9 \pm 3.7$ & $16.1 \pm 3.0$ \\
$3 \mathrm{~d}$ & 8 & $43.0 \pm 5.9$ & $34.6 \pm 10.0$ & 13 & $29.1 \pm 5.2$ & $33.5 \pm 8.7$ \\
$5 \mathrm{~d}$ & 9 & $93.2 \pm 11.3$ & $56.6 \pm 9.8$ & 9 & $64.9 \pm 9.7$ & $58.2 \pm 8.4$ \\
$7 \mathrm{~d}$ & 8 & $62.0 \pm 12.7$ & $54.9 \pm 5.5$ & 8 & $66.3 \pm 10.6$ & $77.3 \pm 10.4$ \\
\hline
\end{tabular}

$N_{C}, N_{E}$ number of control (Ctrl) and regenerating (Exp) discs analyzed, respectively. $P$, posterior; $A$, anterior. 

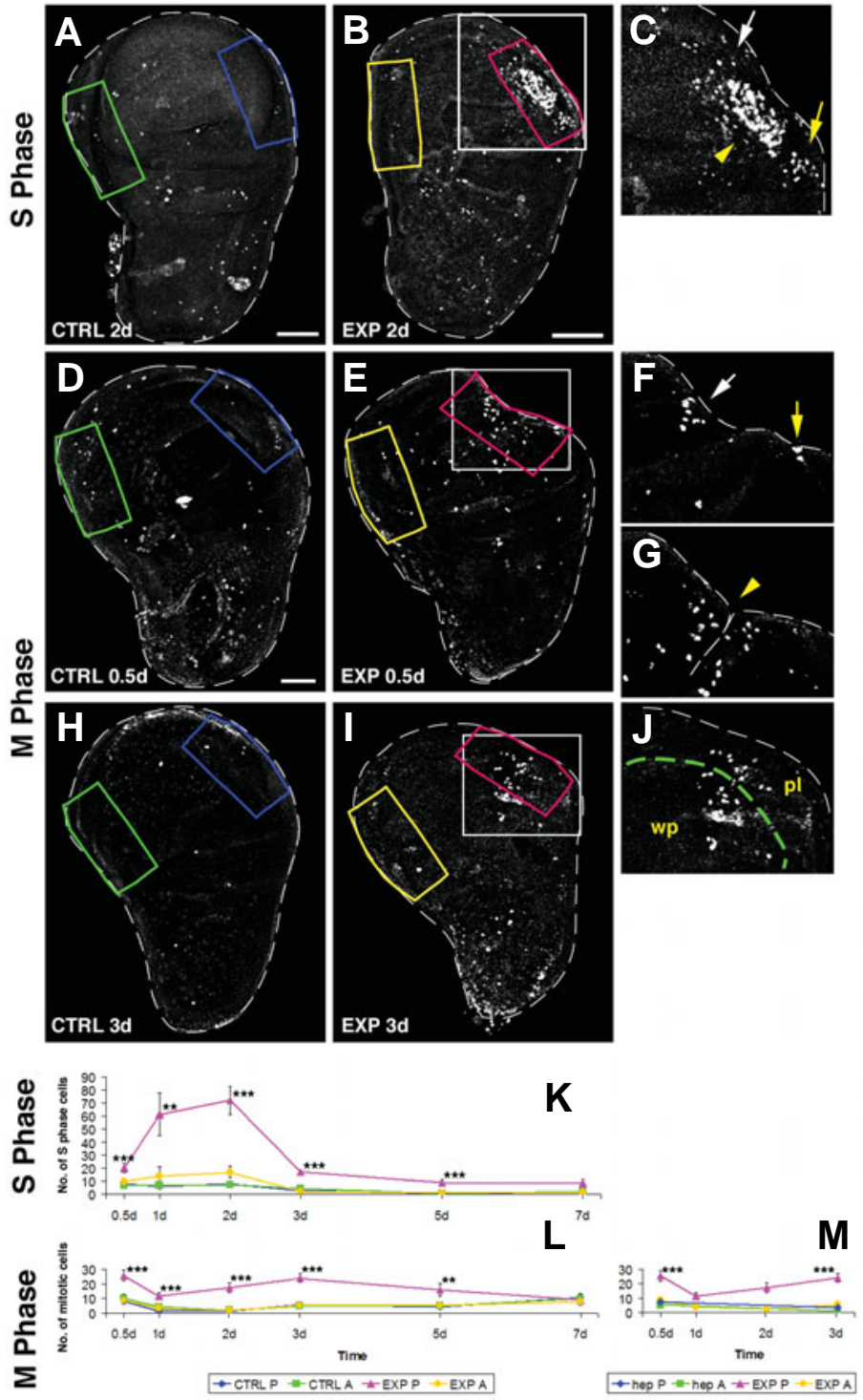

control levels at 7 days, although significant differences between Exp $P$ and the remaining areas were still apparent at 3 and 5 days (Fig. $3 \mathrm{~K}$, Table 1 ). At early stages (0.5 to 1 day), Sphase cells concentrated at the vertex of the wound, while dorsal and ventral folds showed few scattered labeled cells. At 2 days, most $\mathrm{S}$-phase cells were in large clusters, whereas in the folds few scattered labeled cells were still present (Fig. $3 B, C)$. From the third day on, the number of labeled cells decreased steadily, dorsal and ventral folds were no longer recognizable, and S-phase cells were evenly scattered in the regenerated tissue (not shown).

Mitotic cells were analyzed in identical areas to those of BrdU incorporation (Fig. 3D-J, L). At 0.5 days, with the wound still unhealed, the number of mitoses was significantly higher in regenerating areas than in the rest of the disc. At 1 day, mitoses had the lowest values in all areas and resumed again only in Exp $P$ areas, peaking at 3 days. From then on, the number of mitoses decreased, reaching control values at 7 days (Fig. 3L, Table1). At 0.5 and 1 day of regeneration, when wound healing
Fig. 3. Patterns of BrdU incorporation (A-C) and mitosis (D-J) in 3/4A wing disc fragments. $(\mathbf{A}, \mathbf{B})$ Control $(A)$ and experimental $(B)$ discs after 2 days of culture. Areas in anterior $(A)$ and posterior $(P)$ compartments in which BrdU-labelled cells were counted: green (Ctrl A) and blue (Ctrl P) in control discs; yellow (Exp A) and magenta (Exp P) in experimental discs. (C) Higher magnification view of the white box in (B) showing BrdUlabeled cells clustered at the vertex (yellow arrowhead) close to the healed wound (white dashed line) and at the dorsal (yellow arrow) and ventral (white arrow) healing folds. (D,E) Control and experimental discs after 0.5 day of culture. Mitotic cells are mainly concentrated in the wound area (Exp P, magenta box). (F,G) Two confocal planes of the enlarged white square in (E). Mitotic cells are located in the ventral (white arrow) and dorsal (yellow arrow) healing folds and along the edges of the wound (yellow arrowhead). (H,I) Control and experimental discs after 3 days of culture. Mitotic cells are concentrated in the blastema (magenta in I). (J) Higher magnification view of the white box in (I) to show scatterd distribution of mitoses in the blastema. (K) Average number of S-phase labelled cells in each area at different times of regeneration. Significant differences between controls (Ctrl A, Ctrl $P$, and Exp A) and experimental discs (Exp P) are highlighted $\left({ }^{* *} p<0.01,{ }^{* * *} p<0.001\right.$; Table 1). (L) Average number of mitoses in control and experimental areas at different times of regeneration. Exp $P$ values differ significantly from controls and Exp A throughout regeneration ( ${ }^{*} p<0.01,{ }^{* * *} p<0.001$; Table 1). (M) Effect of the allele hemipterous (hep ${ }^{r 75}$ ) on the average number of mitoses at 0.5 and 3 days of regeneration. The number of mitoses in regenerating hep ${ }^{r 75}$ fragments (hep $P$, hep A) was compared to the Exp $P$ and Exp A curves in ( $L$ ), which served as controls. hep $P$ values were significantly lower than those of $\operatorname{Exp} P\left({ }^{* * *} p<0.001\right)$. White dashed lines mark the contour of the discs. In all figures, anterior is to the left and ventral to the top. Scale bar, $50 \mu \mathrm{m}$.

is not yet completed, mitoses were evenly distributed along the wound edges and folds (Fig. 3F, G). At 3 days when the wound is healed and folds are not longer distinguishable, mitoses appeared uniformly distributed in the regenerating area (Fig. $3 \mathrm{H}-\mathrm{J})$.

As JNK signaling plays a central role in wound healing (Bosch et al., 2005, Galko and Krasnow, 2004, Rämet et al., 2002), we tested whether a hypomorphic allele of the Jun amino-terminal kinase kinase hemipterous (hep) (Glise et al., 1995), which keeps the disc unhealed (Bosch et al., 2005), impairs proliferation. Mitoses were measured in anterior (hep A) and posterior (hep $P$ ) areas of hep $\mathrm{p}^{\mathrm{r} 75} / \mathrm{Y}$ regenerating discs at 0.5 and 3 days after cutting and compared to wild type regenerating counterparts (Exp A and Exp P; Fig. 3M). The number of mitoses in hep $A$ and Exp $A$ areas was very low. Interestingly, the number of mitoses in hep $P$ at 0.5 and 3 days was significantly lower than in Exp $P$ areas.

\section{Cell death in regenerating discs}

During the first $12 \mathrm{~h}$ of regeneration, cell death assessed with TUNEL assay was not significantly higher in the Exp $P$ area compared to other areas. The amount of cell death leveled off in all areas at 1-2 days and then increased again throughout the regenerating discs in control and experimental conditions, peaking at 5-7 days (Fig. 4D, Table1). At all time points examined, no significant differences (at $p<0.01$ and $p<0.001$ ) were found between the Exp $P$ area and other areas of the disc (Fig. 4A,B). Therefore, the increasing numbers of dead cells throughout the discs from the third day on is likely to be a response of discs to in vivo culture conditions into female host abdomens. To test whether suppression of apoptosis influ- 

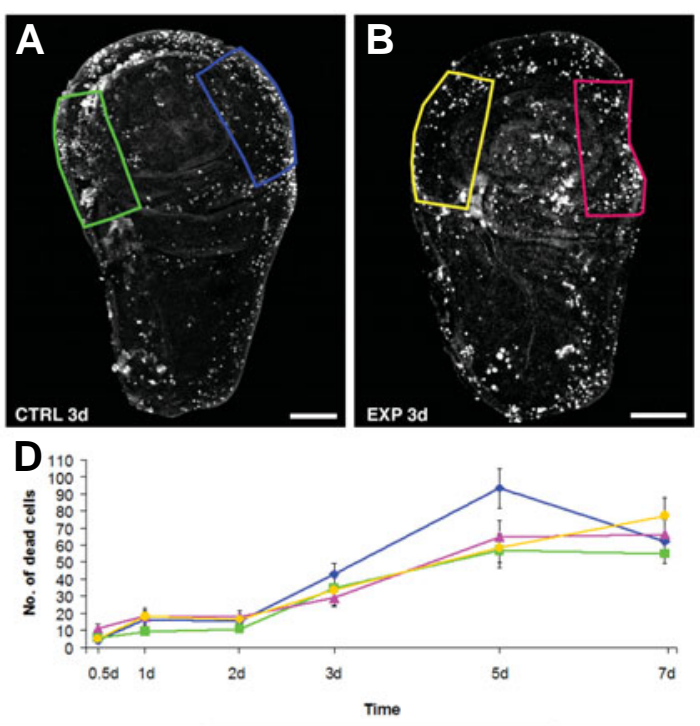

$+C T R L P=C T R L A \neq-\operatorname{XXP}+\operatorname{EXPA}$
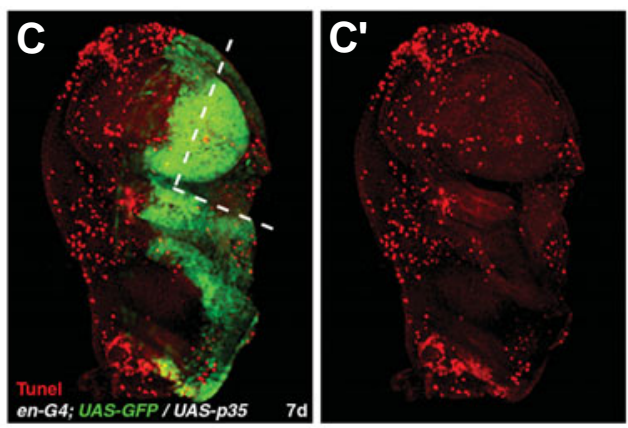

Fig. 4. Cell death assayed with TUNEL staining during regeneration of 3/4A wing disc fragments after various periods of incubation in vivo. (A,B) $A$ disc 3 days after implantation. (C,C') 7 days of regeneration of a disc overexpressing p35 in the posterior (P) compartment driven by en-Gal4 (GFP, green channel). The dashed white line marks the approximate regenerated area. Dead cells are shown in red. Despite cell death being dramatically reduced in the $P$ compartment $\left(C^{\prime}\right)$, regeneration occurs normally. (D) Average number of dead cells in each area at different times of regeneration. Significant differences ( ${ }^{*} p<0.05$; Table 1) were only detected at 5 days between CtrIP and the other areas studied. Scale bar, $50 \mu \mathrm{m} .(C)$ is at same scale as (B).

(WP) contained 15.4 cells $( \pm 2.6$; $n=19)$ whereas clones in the peripheral pleura (PL) 24.5 cells per clone $( \pm 3.7 ; n=13)$. Figure 5 illustrates the topology of clones for the WP and PL areas in blastemas of 22 regenerating discs plotted on a canonical regenerated disc, along with clone shape and orientation. The largest clones in the $\mathrm{PL}$ region were elongated along the periphery of the regenerating disc (6 out of 13), whereas smaller PL clones were mostly isometric. Clones in the WP are mainly elongated (12 out of 19), albeit small, but following the proximo-distal pattern characteristic of clone orientations found in normal development. WP clones near the adjacent $\mathrm{PL}$ also tend to elongate along the WP-PL border. In summary, clones in PL are large and run along the periphery of the

enced regeneration, we activated p35, a suppressor of cell apoptosis, in the posterior compartment using an en-Gal4 driver. Compared to normal levels in the anterior compartment, cell death was almost completely suppressed in the P compartment (Fig. 4C). However, discs still regenerated normally under these conditions.

\section{Clonal analysis}

To characterize the patterns of growth and intercalation in the regenerating tissue, we used the FLP-out technique to stochastically activate Gal4, thereby marking single cells and their descendant clones with UAS-GFP, and analyzed size, shape and topology of clones. Clones in the central wing pouch
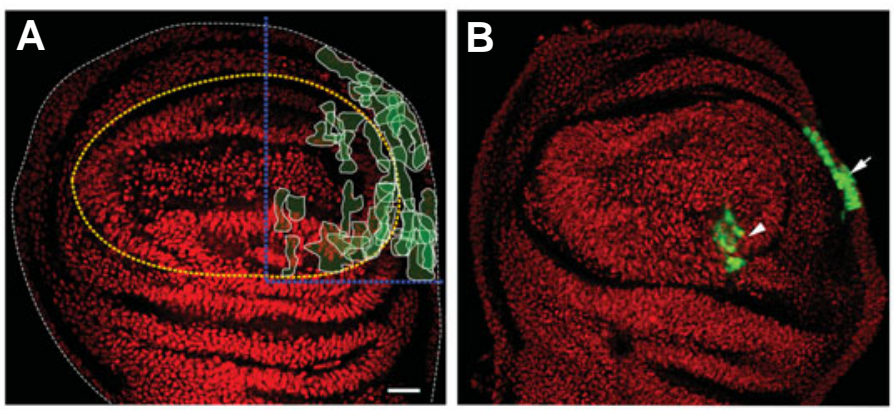

Fig. 5. Clonal analysis of regenerating 3/4A wing discs. (A) Plot of 22 clones on a regenerated disc. Clones in the WP grow preferentially along the proximo-distal axis of the disc. Clones in the PL and clones close to the WP/PL boundary (yellow dashed line) grow preferentially elongated along the periphery of the disc. Blue dashed lines indicate the approximate cut site. White dashed line shows the contour of the disc. (B) Confocal plane of a regenerating disc after 4 days of culture showing a PL elongated clone following the periphery (arrow) and a WP clone following the proximo-distal axis (arrowhead). Notice the absence of clones in the A compartment. In red: nuclei. Scale bar, $20 \mu \mathrm{m}$. disc and clones in the WP are small and grow along the proximo-distal axis, suggesting that differences in mitotic rates and orientations are crucial to re-shape the missing tissue.

\section{Discussion}

In this study, we examined the origin, lineage, and boundaries of the blastema in regenerating wing imaginal discs of Drosophila, along with the temporal and spatial patterns of cell proliferation and the links, as assessed by clonal analysis, between changes in the topology of proliferation and orientation of cell division and the extent of positional disparities. Our results support four main conclusions: (1) cell-lineage tracing methods strongly suggest that blastema cells arise locally from wound cells expressing JNK-in addition, puc expression appears as a good marker to delineate blastema boundaries; (2) DNA synthesis and cell division increase prior to the completion of wound closure along both sides of the wound and are maintained thereafter-hence, wound healing is not a prerequisite for either of those processes; (3) elongated orientation of cell division in the pleura, indicate that larger positional value discontinuities lead to greater intercalary growth; and (4) whereas cell proliferation plays a principal role in blastema formation and growth, cell death seems dispensable in both processes.

\section{The wing disc blastema: boundaries and origin of cells}

In most regenerating systems, blastemas and their boundaries remain well delimited into late stages of regeneration. In imaginal discs, the lack of suitable morphological or molecular markers has precluded clear definition. The sole attempt was that of O'Brochta and Bryant (1987), who defined the blastema in regenerating wing imaginal discs as the area where most S-phase labeled cells cluster. That definition applied to 2-3 days regeneration and the blastema corresponded to an area $25-75 \mu \mathrm{m}$ wide, equivalent to 
8-25 cell diameters from the closed wound. However, in older regenerating discs (e.g. 4-7 days), the extent and limits of the blastema became blurred (Fig. 2 in O'Brochta and Bryant, 1987). Using BrdU we found average widths of $50 \mu \mathrm{m}$ for 2-day-old blastemas (Fig. 3B,C), a value fairly close to that reported by O'Brochta and Bryant (1987). At late stages, the JNK phosphatase puckered (puc) served as a useful marker to define late blastema boundaries. puc is activated in 10-15 rows of cells from the wound edges at $5 \mathrm{~h}$ of regeneration, decreases from $24 \mathrm{~h}$ onwards, when the wound is healed, and is still faintly detectable later on (Bosch et al., 2005). This is the first report of a useful marker for early blastema boundaries in regenerating imaginal wing discs, despite its resolution being somewhat limited.

If cells containing activated puc at the early wound margin mark late blastema boundaries, it follows that most blastema cells should stem from those early cells. Indeed, most cells in regenerated tissue (Fig. 1) are lineage tagged with lacZ activated by pucGal4. Further evidence comes from the percentages of puc-GFPpositive and puc-GFP-negative mitotic cells within the blastema. Whereas in $12 \mathrm{~h}$ blastemas puc-GFP-positive mitotic cells reach $60 \%$, this percentage dwindles to a mere $15 \%$ at 7 days (Fig. $1 \mathrm{H})$. This indicates that while a substantial number of cells at the wound margin are puc-positive, others are puc-negative. These puc-negative cells might well be derivatives of cells or groups of cells containing little or no GFP. Furthermore, during blastema growth, new cells are progressively displaced by intercalary growth outside the JNK-signaling domain. These cells switch off GFP expression, and this accounts for the decreasing percentages of GFP-positive mitotic cells (Fig. 1H). Finally, the presence of cells in mitosis in the early wound area as late as 7 days of regeneration (yellow and red dots in the GFP belt; Fig. 1G) strongly suggests that pattern restoration involves a larger than expected area of the regenerating fragment.

In addition, we conclude that both edges of the blastema contribute to the regeneration of the missing tissue because aplabeled cells regenerate exclusively the dorsal part of the blastema. This also indicates that dorsal compartment cells retain their dorsal identity during regeneration. It has been previously found that clones induced prior to cutting were able to cross compartment boundaries in the wing disc, but only when clones were generated in Minute heterozygous mutant background to confer proliferative advantage to the fast growing clone (Szabad et al., 1979).

\section{The wing disc blastema: growth dynamics}

Our results confirm previous studies (see Introduction) indicating that the response of wing imaginal discs to wounding includes the local stimulation of DNA synthesis in cells close to the wound prior to completion of wound healing. However, in agreement with the findings of Kiehle and Schubiger (1985) and Mattila et al. (2005), but in contrast to most previous reports, we also detected a significant increase in mitosis prior to wound healing. Therefore, homotypic healing of the wound edges is not a prerequisite to enhance either DNA synthesis or mitosis. Nevertheless, later on wound healing may be a necessary requirement for regeneration. Mutation of the JNK signaling pathway disrupts wound healing and regeneration in wing disc fragments (Bosch et al., 2005) and the number of mitoses in regenerating fragments is strongly decreased (Fig. 3M; Mattila et al., 2005). How JNK signaling is activated at the wound edges of regenerating wing imaginal discs is unknown. While it could be postulated to be due to the release of growth factors or to changes in the extracellular matrix, etc, activation of JNK signaling due to loss of cell polarity (Igaki et al., 2006) and/or to mechanical stresses at the contracting wound edge (Nelson et al., 2005) also merits further study.

Temporal patterns of S-phase cells and mitotic cells in regenerating blastemas were similar (Figs. 3K,L). As expected, maximum mitosis lagged one day behind the peak in the number of $S-$ phase cells and the overall number of mitoses was much lower than the number of S-phase cells. From 3 days on, however, the slope of the mitotic curve had a slower decrease than that of $S$ phase, so that overall numbers for mitotic and S-phase cells leveled off at 5 days. The topography of S-phase and mitotic cells within the regenerative blastema is important in terms of the model of epimorphic regeneration (O'Brochta and Bryant, 1987). Sequential models predict that growth will be highly localized throughout regeneration, while averaging models predict that growth will be localized at first, that the growth zone (the blastema) will expand during regeneration, and that at later stages proliferating cells will be distributed throughout the blastema. In agreement with previous reports (Bryant and Fraser, 1988, Dale and Bownes, 1980, O'Brochta and Bryant, 1987), we found that cell proliferation is highly localized in the region adjacent to the wound during the first two days of regeneration. Later, it is found to be scattered. Also, total number and frequency of clones increase, as expected, as regeneration proceeds. Taken together, these data support the averaging models, indicating that proliferation occurs throughout the blastema, and therefore, that growth is intercalary.

Intercalary growth depends on discrepancies in positional values between neighboring cells, leading to differential cell proliferation (Bohn, 1974). Thus, we anticipated that the number of cells added by proliferation to restore the original pattern in $3 /$ $4 \mathrm{~A}$ regenerating disc fragments would be related to the positional disparity between apposed surfaces after wound healing. Such disparities are likely to be smaller at the WP than in the peripheral $\mathrm{PL}$ area. Therefore, we expected PL clones, which have to fill a long and thin stretch of tissue, would be larger and preferentially oriented along the periphery than WP clones. Indeed, our clonal analysis shows that the largest clones are elongated and peripherally oriented in the PL. However, as in the regenerating 3/4A fragment, clones in the developing wing grow along the proximodistal axis when fall in the WP and along the periphery when fall in the PL (Baena-López et al., 2005, Garcia-Bellido and Merriam, 1971, Resino et al., 2002). It has been proposed that during wing development, preferential orientations of cell division along axes of growth and lineage boundaries are linked to positional disparities and, hence, to final organ and tissue shape (Baena-López et al., 2005). Therefore, an alternative view will be that growth processes during regeneration of the $3 / 4 \mathrm{~A}$ fragments and during imaginal disc development are both driven to smoothen the disparities in positional values.

Taken together, our results suggest that pattern formation during regeneration in wing imaginal discs is the result of the concurrent effects of heterogeneities in both cell proliferation and preferred orientations of cell division among different areas of the disc. Such heterogeneities may, in turn, be due to cells reading local differences in the expression of key molecules. The nature 
of these molecules and how cells integrate their signals to elicit oriented cell division are still poorly understood.

\section{Materials and Methods}

\section{Drosophila stocks and genetics}

A description of genetic markers and chromosome balancers used in this study can be found in FlyBase. Fly cultures and crosses were grown on standard fly medium at $25^{\circ} \mathrm{C}$. Analysis of cell proliferation and cell death were performed using Canton $\mathrm{S}$ as a wild type stock. hep ${ }^{75}$ (Glise et al., 1995) was used as a JNK mutant and UAS-GFP;puc E69-A-Gal4 (Pastor-Pareja et al., 2004) as a marker of JNK activity.

\section{In vivo disc culture and microsurgery}

Imaginal-disc manipulation was performed as described previously (Bosch et al., 2005). Wing imaginal discs were removed from mid-to-late third instar larvae, 100-120 $\mathrm{h}$ after egg laying (AEL), into Schneider's insect medium (Sigma). A $90^{\circ}$ sector was dissected out using tungsten needles from the posterior $(P)$ compartment, leaving a $3 / 4$ anterior $(3 / 4 A)$ fragment. Experimental (3/4A fragments) and control (uncut) discs were implanted into recently eclosed Canton $\mathrm{S}$ females and kept at $25^{\circ} \mathrm{C}$. Following a culture period of $12 \mathrm{~h}$ or 1, 2, 3, 4, 5 and 7 days, implants were removed from the hosts and analyzed. Implants with unclear morphologies were discarded. For each experiment and time point, a minimum of 8 discs were used for further analyses.

In all experiments presented here, wound healing was completed after $24 \mathrm{~h}$ of incubation, as described previously (Bosch et al., 2005). Briefly, during healing of the type of cut described above, the two wound edges meet together and wound closes in a zipper-like mode starting from the vertex of the cut area (Fig1 A) and zippering the rest of the edges of the tissue. By the end of closure, two folds can remain visible at the proximal region, one corresponding to the most dorsal wound edge and another to the ventral edge, and therefore named dorsal and ventral fold respectively. The presence of these folds during early stages of regeneration together with the molecular markers used in this work (GFP tagged constructs, see below) helped us to precisely recognize and define the wound edges and regenerating areas of the discs.

\section{Cell lineage experiments}

Cell lineage of regeneration blastemas was followed in wing discs of two different genotypes: UAS-GFP/Act5c $>$ stop $>$ lacZ; puC E69-A_Ga/4/UASFLPand Act5c-FRT-stop-FRT-lacZ/ap-Gal4UAS-GFP;UAS-FLP. To study the cell lineage of the puc and ap domains, wing discs were cut and the 3/4A fragment kept (Fig. 4A-B', 7B-C'). All fragments were implanted inside adult hosts and left to regenerate. To detect lacZ expression, an anti- $\beta$-galactosidase antibody (1:1000, Cappel) was used and was detected with a donkey anti-rabbit Rhodamine Red-labeled secondary antibody (1:200, Jackson Immunoresearch).

\section{BrdU incorporation labeling of mitosis and cell death}

Control and regenerating implants were removed from adult hosts, incubated in $100 \mu \mathrm{g} / \mathrm{ml}$ bromodeoxyuridine (BrdU) in Schneider's insect medium for 30 min and fixed in 4\% paraformaldehyde in PBS for 20 min. After washing in PBS, they were frozen in methanol at $-20^{\circ} \mathrm{C}$ for $30 \mathrm{~min}$. Implants were then washed in PBT (PBS+0.3\% Triton X100), hydrolyzed for 45 min with $\mathrm{HCl} 2 \mathrm{~N}$, and washed again in PBT. Incorporated BrdU was localized using a monoclonal mouse anti-BrdU (1:20; Beckton and Dickinson) and a Rhodamine Red-conjugated goat anti-mouse secondary antibody (1:200, Jackson Immunoresearch), using a standard immunohistochemistry protocol.

Control and regenerating implants were removed from adult hosts and immunostained using anti-PhosphoHistone3 ( $\mathrm{PH} 3,1: 1000$, Upstate Biotechnology) and a Rhodamine Red-conjugated donkey anti-rabbit secondary antibody (1:200, Jackson Immunoresearch). Imaginal discs were mounted in SlowFade Light Antifade (Molecular Probes, Inc) prior to confocal analysis (Olympus Flouview 500).

To label dead cells with TUNEL assay, control and regenerating implants were removed from adult hosts and fixed for 20 min in 4\% paraformaldehyde in PBS, permeabilized in PBT for $30 \mathrm{~min}$, and incubated with terminal deoxynucleotidyl transferase (Roche) in a reaction mix containing Chromatide BODIPY® Texas Red-14-dUTP (Molecular Probes, Inc) for $1 \mathrm{~h} 30 \mathrm{~min}$ at $37^{\circ} \mathrm{C}$. After washes, discs were mounted in SlowFade Light Antifade (Molecular Probes, Inc). Wing discs of the genotype w; en-Gal4/CyO; UAS-GFP/UAS-p35 were used to inhibit apoptosis in the $\mathrm{P}$ compartment.

\section{Image analysis and statistics}

Labeled cells were counted in confocal projections from areas of a similar size in the $\mathrm{P}$ and $\mathrm{A}$ compartments. These areas roughly correspond to $10 \%$ of the total area of each disc. In control uncut discs they were named Control $P(C \operatorname{trl} P)$ and Control $A(C t r l A)$, and in experimental cut discs Exp $P$ and Exp $A$. In unhealed fragments ( 0 and $12 \mathrm{~h}$ ), the Exp $P$ areas were outlined following the edge of the wound and covering an area equal to control and healed discs. Drawing and cell counting were done using ImageJ software (NIH Image; www.rsb.info.nih.gov/ij).

An average comparison test was used for statistical analysis. Differences were considered statistically significant at $p<0.01$ and $p<0.001$.

\section{Clonal analysis}

Clones were generated in larve of the hs-FLP 1.22;+;Act5c-FRT CD2 FRT-Gal4 UAS-GFP genotype. Adult hosts, bearing regenerating implants, were heat-shocked for $20 \mathrm{~min}$ at $29^{\circ} \mathrm{C}$ at $2 \mathrm{~h}$ after implantation, and regenerating discs removed at 4 days of regeneration for clonal analysis. In all discs, clones were outlined over confocal planes of regenerated discs, stained for nuclei to count the number of cells, plotted, and drawn (see Fig. 5A,B). Clone outlines and plots were prepared using Freehand 9.0 software (Macromedia, Inc). Nuclei were stained using Sytox Orange (1:20000; Molecular Probes, Inc)

As controls, 4-day regenerating discs $(n=8)$ implanted into non-heatshocked adult hosts were used to estimate the number of spontaneous clones due to leakage of the flipase after incubation of host flies at $25^{\circ} \mathrm{C}$. Since these spontaneous clones contained 1-10 cells, we have used clones larger than 10 cells per clone in the induced discs for further analysis. The number of cells per clone (clone size) in regenerating discs was measured in 2 areas of the $P$ compartment (i.e. cut and regenerated domain): the wing pouch (WP) and the ventro-lateral pleura-hinge region $(\mathrm{PL})$. Clone sizes were determined by counting the number of GFPlabeled cells in each clone and expressed as the mean number of cells per clone. An average comparison test was used for statistical analysis. Differences were considered statistically significant at $p<0.05$.

\section{Acknowledgments}

We thank M. Corominas for her valuable comments throughout the work. We also thank E. Martin-Blanco for comments on the manuscript and $L$. Serra for help with statistical analysis. $M B$ was a recipient of a predoctoral grant from the University of Barcelona. JB and FS were funded by grants from DGICYT, Ministerio de Ciencia y Tecnología, and the Generalitat de Catalunya.

\section{References}

ADLER, P.N. (1984). DNA replication and pattern regulation in the imaginal wing disc of Drosophila. Dev Biol102: 300-8.

BAENA-LÓPEZ, L.A., BAONZA, A. and GARCÍA-BELLIDO, A. (2005). The orientation of cell divisions determines the shape of Drosophila organs. Curr Bio/15: 1640-4.

BOHN, H. (1974). Extent and properties of the regeneration field in the larval legs of cockroaches (Leucophaea maderae) iii. Origin of the tissues and determination of symmetry properties in the regenerates. J Embryo/ Exp Morpho/32: 8198. 
BOSCH, M., SERRAS, F., MARTIN-BLANCO, E. and BAGUNA, J. (2005). Jnk signaling pathway required for wound healing in regenerating Drosophila wing imaginal discs. Dev Bio/280: 73-86.

BRYANT, P.J. and FRASER, S.E. (1988). Wound healing, cell communication, and DNA synthesis during imaginal disc regeneration in Drosophila. Dev Bio/127: 197-208.

BRYANT, P.J. and O'BROCHTA, D.A. (1986). Growth patterns in Drosophila imaginal discs. Prog Clin Biol Res 217A: 297-300.

DALE, L. and BOWNES, M. (1980). Is regeneration in Drosophila the result of epimorphic regulation? Wilhelm Roux's Archieves 189: 91-96.

DALE, L. and BOWNES, M. (1981). Wound healing and regeneration in the imaginal disc of Drosophila. Wilhelm Roux's Archieves 190: 185-190.

FAIN, M.J. and ALVAREZ, C. (1987). The cell cycle and its relation to gowth during pattern regulation in wing discs of'Drosophila. J. Insect. Physiol. 33: 697-706.

FRENCH, V., BRYANT, P.J. and BRYANT, S.V. (1976). Pattern regulation in epimorphic fields. Science 193: 969-81.

GALKO, M.J. and KRASNOW, M.A. (2004). Cellular and genetic analysis of wound healing in Drosophila larvae. PLoS Biol 2: E239.

GARCIA-BELLIDO, A. and MERRIAM, J. (1971). Parameters of the wing imaginal disc development of Drosophila melanogaster. Dev Bio/24: 61-87.

GLISE, B., BOURBON, H. and NOSELLI, S. (1995). Hemipterous encodes a novel Drosophila map kinase kinase, required for epithelial cell sheet movement. Cell 83: 451-61.

IGAKI, T., PAGLIARINI, R.A. and XU, T. (2006). Loss of cell polarity drives tumor growth and invasion through JNK activation in Drosophila. Curr Bio/16: 113946.

KIEHLE, C.P. and SCHUBIGER, G. (1985). Cell proliferation changes during pattern regulation in imaginal leg discs of Drosophila melanogaster. Dev Biol 109: 336-46
LEE, N., MAURANGE, C., RINGROSE, L. and PARO, R. (2005). Suppression of polycomb group proteins by JNK signaling induces transdetermination in Drosophila imaginal discs. Nature 438: 234-7.

MATTILA, J., OMELYANCHUK, L., KYTTALA, S., TURUNEN, H. and NOKKALA, S. (2005). Role of jun n-terminal kinase (JNK) signaling in the wound healing and regeneration of a Drosophila melanogaster wing imaginal disc. Int J Dev Bio/49: $391-9$

MORGAN, T.H. (1901). Regeneration. Macmillan, London.

NELSON, C.M., JEAN, R.P., TAN, J.L., LIU, W.F., SNIADECKI, N.J., SPECTOR, A.A. and CHEN, C.S. (2005). Emergent patterns of growth controlled by multicellular form and mechanics. Proc Natl Acad Sci U S A 102: 11594-9.

O'BROCHTA, D.A. and BRYANT, P.J. (1987). Distribution of s-phase cells during the regeneration of Drosophila imaginal wing discs. Dev Biol119: 137-42.

PASTOR-PAREJA, J.C., GRAWE, F., MARTIN-BLANCO, E. and GARCIA-BELLIDO, A. (2004). Invasive cell behavior during Drosophila imaginal disc eversion is mediated by the jnk signaling cascade. Dev Cel/7: 387-99.

RÄMET, M., LANOT, R., ZACHARY, D. and MANFRUELLI, P. (2002). Jnk signaling pathway is required for efficient wound healing in Drosophila. Dev Bio/241: 14556.

RESINO, J., SALAMA-COHEN, P. and GARCÍA-BELLIDO, A. (2002). Determining the role of patterned cell proliferation in the shape and size of the Drosophila wing. Proc Natl Acad Sci U S A 99: 7502-7.

SZABAD, J., SIMPSON, P. and NOTHIGER, R. (1979). Regeneration and compartments in Drosophila. J Embryol Exp Morpho/49: 229-41.

WEIGMANN, K. and COHEN, S.M. (1999). Lineage-tracing cells born in different domains along the pd axis of the developing Drosophila leg. Development 126: 3823-30.

WOLPERT, L. (1969). Positional information and the spatial pattern of cellular differentiation. J Theor Biol25: 1-47.

\section{Related, previously published Int. J. Dev. Biol. articles}

See our recent Special Issue Fertilization, in honor of David L. Garbers and edited by Paul M. Wassarman and Victor D. Vacquier at: http://www.ijdb.ehu.es/web/contents.php?vol=52\&issue=5-6

See our recent Special Issue Ear Development edited by Fernando Giraldez and Bernd Fritzsch at: http://www.ijdb.ehu.es/web/contents.php?vol=51\&issue=6-7

Apoptosis in Drosophila: compensatory proliferation and undead cells Francisco A. Martín, Ainhoa Peréz-Garijo and Ginés Morata Int. J. Dev. Biol. (2008) 52: 2447-2447

Role of Jun N-terminal Kinase (JNK) signaling in the wound healing and regeneration of a Drosophila melanogaster wing imaginal disc

Jaakko Mattila, Leonid Omelyanchuk, Satu Kyttälä, Heikki Turunen and Seppo Nokkala

Int. J. Dev. Biol. (2005) 49: 391-399

Dynamics of decapentaplegic expression during regeneration of the Drosophila melanogaster wing imaginal disc Jaakko Mattila, Leonid Omelyanchuk and Seppo Nokkala Int. J. Dev. Biol. (2004) 48: 343-347

Distinctive expression of Myf5 in relation to differentiation and plasticity of newt muscle cells

Yutaka Imokawa, Phillip B. Gates, Young-Tae Chang, Hans-Georg Simon and Jeremy P. Brockes

Int. J. Dev. Biol. (2004) 48: 285-291

2006 ISI ${ }^{* *}$ Impact Factor $=3.577^{\star *}$

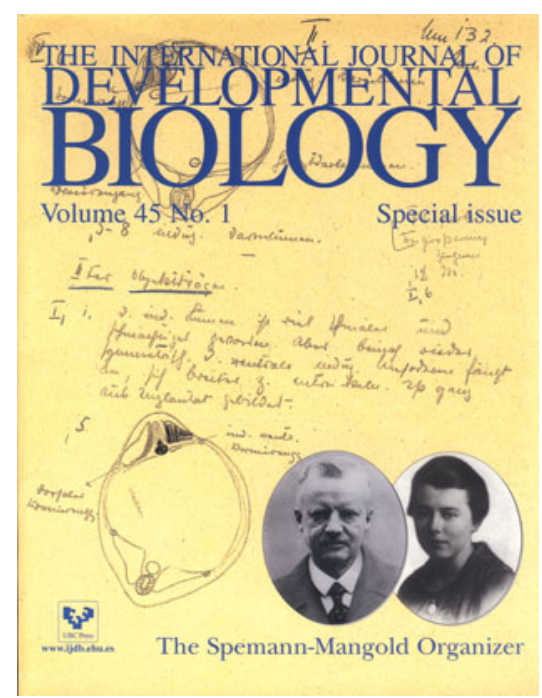

